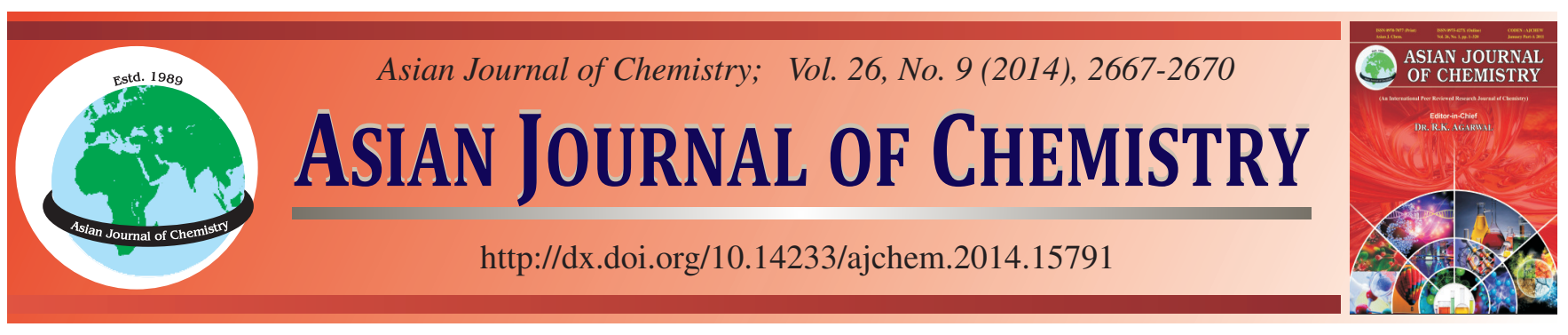

\title{
A Novel Mutually Perpendicular Bicyclo-Shape Tetranuclear Complex Constructed from 2,3-Dihydroxy butanedioic Acid with Pisces Mode
}

\author{
L. JIANG ${ }^{1,2}$, B.Q. YANG ${ }^{1, *}$ and G.D. FENG ${ }^{2}$
}

${ }^{1}$ College of Chemistry and Chemical Engineering, Shaanxi University of Science and Technology, Xi'an 710021, P.R. China

${ }^{2}$ Key Laboratory of Functional Coordination Compounds, Department of Chemistry and Chemical Engineering, Baoji University of Arts and Science, Baoji 721013, P.R. China

*Corresponding authors: E-mail: ginger_j1@163.com; fengguodong00805@163.com

Received: 17 May 2013;

Accepted: 3 September 2013;

Published online: 28 April 2014;

AJC-15093

A new complex $\left[\mathrm{Ni}_{4}(\mathrm{dhbd})_{4} \cdot 8 \mathrm{H}_{2} \mathrm{O}\right] \cdot 13 \mathrm{H}_{2} \mathrm{O}(\mathbf{1})\left(\mathrm{H}_{4} \mathrm{dhbd}=2,3\right.$-dihydroxy butanedioic acid $)$ has been synthesized and its structure $\left(\mathrm{C}_{16} \mathrm{H}_{16} \mathrm{O}_{45} \mathrm{Ni}_{4}\right.$, $\mathrm{Mr}=1163.13)$ was determined by single-crystal X-ray diffraction analysis. The crystal belongs to the monoclinic system, space group $\mathrm{P}_{21 / \mathrm{c}}$ with $\mathrm{a}=13.700(5), \mathrm{b}=17.143(5), \mathrm{c}=18.599(5) \AA, \beta=96.786(5), \mathrm{V}=4338(2) \AA^{3}, \mathrm{Z}=4, \mathrm{Dc}=1.781 \mathrm{~g} / \mathrm{cm}^{3}, \mu\left(\mathrm{MoK}_{\alpha}\right)=1.839 \mathrm{~mm}^{-1}, \mathrm{~F}(000)$ $=2336, \mathrm{~S}=1.025$, the final $\mathrm{R}=0.0524$ and $\mathrm{wR}=0.1390$ for 9257 reflections with $\mathrm{I}>2 \sigma(\mathrm{I})$. The centrosymmetric nickel complex contains a tetranuclear in which four distorted octahedral $\mathrm{Ni}$ (II) centers are bridged by the carboxylic oxygen atoms and the hydroxy oxygen atoms of the four 2,3-dihydroxy butanedioic acid with the Pisces mode, featuring a novel mutually perpendicular bicyclo structure. The complex units are further linked to form three-dimentional supramolecular structure via the hydrogen bonds between the coordinate water molecules and the plentiful free water molecules. The elemental analysis and optical properties for the complex have been studied.

Keywords: Synthesis, Crystal structure, 2,3-Dihydroxybutanedioic Acid, Bicyclo structure, Nickel(II) complex.

\section{INTRODUCTION}

During recent years, the topological mimicking of natural minerals and constructions of novel complexes with new topologies have been extensively investigated not only for the fundamental interest to reveal the intriguing structural diversity and assembling processes of these materials but also for the pragmatic perspective to obtain new functional materials ${ }^{1-4}$. Recent reports indicate that di-/poly-carboxylate ligands have been proved to be a powerful tool in constructing well-ordered multidimensional supramolecular architectures. Owing to their potential applications as zeolite-like solids in catalysis, the use of hydrogen-bond in the ligands as mentioned above to construct supramolecular frameworks with large porosity and channel has become an appealing field ${ }^{5-7}$. Among these carboxylate-containing ligands, the 2,3-dihydroxy butanedioic acid ligand possesses two terminal carboxyl groups and two hydroxyl groups that may be completely or partially deprotonated, inducing rich coordination quomodos and allowing captivating structures with higher dimensions ${ }^{8}$. Meanwhile, 2,3-dihydroxybutanedioic acid can act as not only hydrogen bond acceptors but also as hydrogen bond donors, depending on the different number of deprotonated hydroxyl and carboxyl groups, to form diverse new extended structures by means of additional hydrogen-bond interactions ${ }^{9}$. Herein we report the synthesis and characterization of a novel mutually perpendicular bicyclo-shape tetranuclear supramolecular framework $\left[\mathrm{Ni}_{4}(\mathrm{dhbd})_{4} \cdot 8 \mathrm{H}_{2} \mathrm{O}\right] \cdot 13 \mathrm{H}_{2} \mathrm{O}$, constructed from 2,3-dihydroxy butanedioic acid $\left(\mathrm{H}_{4} \mathrm{dhbd}\right)$ ligand with unusual Pisces mode.

\section{EXPERIMENTAL}

All chemical reagents were of analytical grade and used without further purification. $\mathrm{C}, \mathrm{H}$ and $\mathrm{N}$ analyses were made on Elementar Vario EL-III elemental analyzer. FT-IR spectra were recorded in the range $4000-400 \mathrm{~cm}^{-1}$ on a Nicolet Avatar360 spectrometer using a KBr pellet.The single-crystal structure of the compound $\mathbf{1}$ was determined on a Bruker APXII CCD diffractometer.

The complex $\left[\mathrm{Ni}_{4}(\mathrm{dhbd})_{4} \cdot 8 \mathrm{H}_{2} \mathrm{O}\right] \cdot 13 \mathrm{H}_{2} \mathrm{O}(\mathbf{1})$ was prepared by the mixture of $\mathrm{Ni}\left(\mathrm{NO}_{3}\right)_{2} \cdot 6 \mathrm{H}_{2} \mathrm{O}(0.145 \mathrm{~g}, 0.5 \mathrm{mmol}), 2,3-$ dihydroxy butanedioic acid $(0.0750 \mathrm{~g}, 0.5 \mathrm{mmol})$ and $\mathrm{H}_{2} \mathrm{O}$ $(16 \mathrm{~mL})$ was heated at $170{ }^{\circ} \mathrm{C}$ for 3 days in a $25 \mathrm{~mL}$ Teflonlined stainless steel vessel under autogenous pressure. After the reaction mixture was slowly cooled down to room temperature, colourless block crystals were obtained. Yield: $76 \%$. For $\mathrm{C}_{16} \mathrm{H}_{16} \mathrm{O}_{45} \mathrm{Ni}_{4}$ Anal. calc. \%: C 16.50; H 1.38. Found (\%): C 17.65; H 1.51. 
X-ray structure determination: A blue colour block single crystal of complex 1 with $0.42 \mathrm{~mm} \times 0.31 \mathrm{~mm} \times 0.21$ $\mathrm{mm}$ was carefully selected under a polarizing microscope and were mounted on a glass fiber and used for X-ray diffraction analyses. Single crystal structure determination by X-ray diffraction measurements were performed using a Bruker APXII CCD diffractometer with graphite monochromated $\mathrm{MoK}_{\alpha}(\mathrm{k}=0.71069 \AA)$ radiation in the range of $1.50 \leq \theta \leq$ $28.31^{\circ}(-17<=\mathrm{h}<=18,-22<=\mathrm{k}<=19,-23<=1<=20)$ at $293 \mathrm{~K}$. Absorption corrections were applied using the multiscan technique ${ }^{10}$. A total of 24913 reflections including 9839 unique ones were collected, of which 9257 with $\mathrm{I}>2 \sigma(\mathrm{I})$ were considered as observed and used in the succeeding refinements. The structure was solved by the Direct Method and refined by full-matrix least-square techniques on $\mathrm{F}^{2}$ using SHELXL-97 ${ }^{11}$. All of the non-hydrogen atoms were refined anisotropically ${ }^{12}$. The $\mathrm{H}$ atoms attached to $\mathrm{C}$ atoms were positioned geometrically, with Uiso values derived from Ueq values of the corresponding $\mathrm{C}$ atom. The final $\mathrm{R}=0.0524$ and $\mathrm{w}=0.1390, \mathrm{~S}=$ $1.025,(\Delta \rho)_{\max }=1.649$ and $(\Delta \rho)_{\min }=-1.230 \mathrm{e} / \AA$ 3 3 .

Crystallographic data for the structural analysis(es) have been deposited with the Cambridge Crystallographic Data Centre, CCDC No.: 908416 for the complex 1.

\section{RESULTS AND DISCUSSION}

The single crystal X-ray analysis showed that complex $\mathbf{1}$ crystallized in monoclinic, space group $\mathrm{P}_{21 / \mathrm{c}}$. The asymmetrical unit of complex 1 contains four $\mathrm{Ni}^{2+}$ ion, four dhbd ligand, eight coordinate water and thirteen free water molecules. As shown in Fig. 1, the Ni ion shows a distorted octahedral coordination geometry with four oxygen atoms from the carboxylate and the adjacent hydroxy groups in two different dhbd ligands

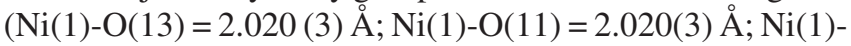
$\mathrm{O}(12)=2.068(3) \AA \circ$ i $(1)-\mathrm{O}(9)=2.080(3) \AA$ ) and two oxygen atoms of the two coordinate water $(\mathrm{Ni}(1)-\mathrm{O}(15)=2.103(4)$ $\AA$; Ni(1)-O(14) = 2.059(4) ̊). The Ni-O (2.007(3)-2.108(3) $\AA$ ) bond distances are all in the normal range. Each dhbd anion acts as a $\mu_{2}$-bridge with the carboxylate and the adjacent
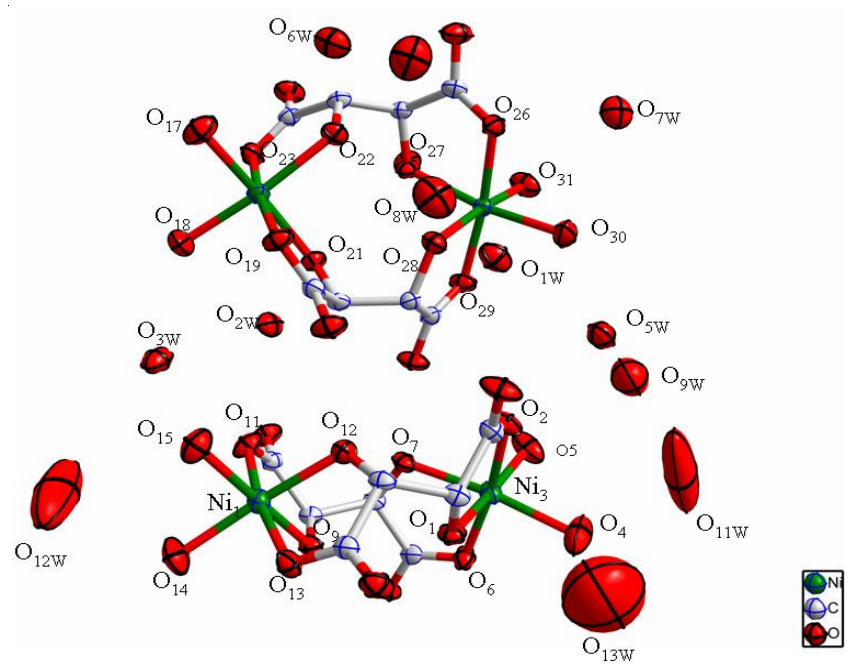

Fig. 1. Coordination geometry of $\mathrm{Ni}(\mathrm{II})$ atom in complex $\mathbf{1}$. Thermal ellipsoids are drawn at $50 \%$ probability and hydrogen atoms were omitted for clarity. Colour codes: Colour codes: $\mathrm{Ni}$, green; C, gray; $\mathrm{O}$, red hydroxy groups in the chelate coordination mode, in which the carboxylate groups exhibit a $\mu^{1}-\eta^{1}: \eta^{0}$ coordination mode ${ }^{13}$. Bidentate-chelating through the dhbd anion, the Ni(II) ions are connected to form the two 12-membered ring, which featuring a novel mutually perpendicular bicyclo-shape (Fig. 2) and the $\mathrm{Ni} \cdots \mathrm{Ni}$ separation through $\mathrm{H}_{2}$ dhbd chelate anions are 5.0123(13) $\AA$ and 5.0680(13) $\AA$, respectively.
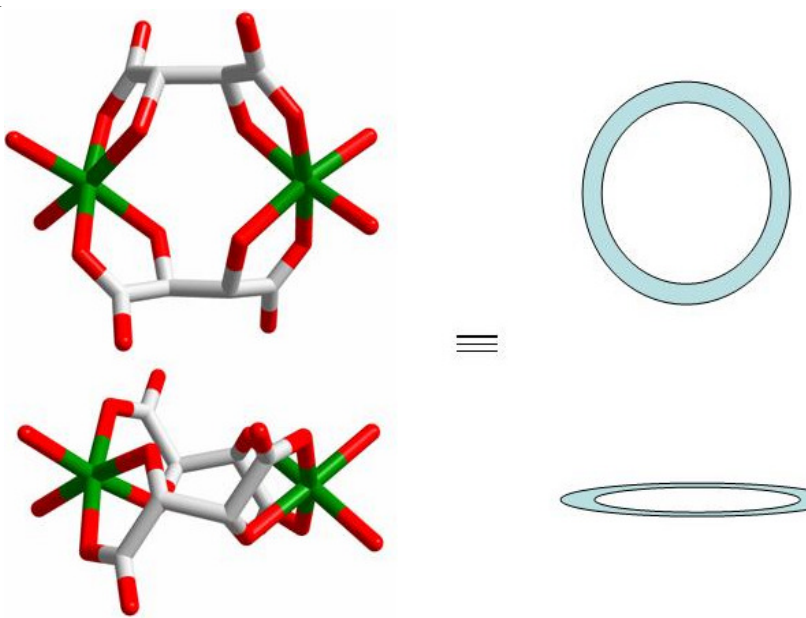

Fig. 2. Scheme of the mutually perpendicular bicyclo-shape. Colour codes: Colour codes: $\mathrm{Ni}$, green; $\mathrm{C}$, gray; $\mathrm{O}$, red

As a result, the mutually perpendicular bicyclo is further linked to a 3D supramolecular structure by the intermolecular hydrogen bonds between the free water molecules and the uncoordinated carboxylate from the dhbd anion $\left(\mathrm{O}_{(4 \mathrm{~W})^{-}}\right.$ $\mathrm{H}_{(4)} \ldots \mathrm{O}_{(20)}=2.792(5) \AA, \mathrm{O}_{(6 \mathrm{~W})}-\mathrm{H}_{(7)} \ldots \mathrm{O}_{(32)}{ }^{\mathrm{iii}}=2.765(5) \AA$, ${ }^{\mathrm{iii}}=$ $1-\mathrm{x}, 1 / 2+\mathrm{y}, 1 / 2-\mathrm{z})$. Moreover, as the multiple intermolecular hydrogen bond acceptors and donors $\left(\mathrm{O}_{(1 \mathrm{~W})}-\mathrm{H}_{(1)} \ldots \mathrm{O}_{(8 \mathrm{~W})}=\right.$ 2.720(6) $\left.\AA ; \mathrm{O}_{(8 \mathrm{~W})}-\mathrm{H}_{(8)} \ldots \mathrm{O}_{(4 \mathrm{~W})}=2.813(6) \AA\right)$, the excessive water molecules interacting with the complex from the bicyclo above and below, is shown in Fig. 4, play an important role in the formation of the 3D supramolecular architecture and stabilize the crystal structure. In addition, there are still abundant hydrogen bonds formed by not only water molecules but also hydroxyl group of dhbd ligands in $\mathbf{1}$ (Table-2). The most striking feature of complex 1 is that, as show in Fig. 4, the 12membered ring is constructed by the two dhbd ligands with the end to end mode, similer as the Pisces pattern (Fig. 3).

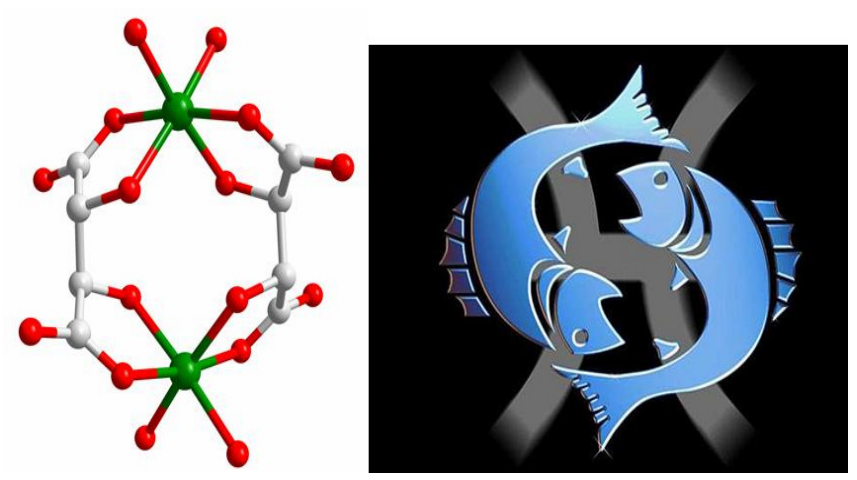

Fig. 3. Illustration of the 12-membered ring with the end to end mode, similer as the Pisces pattern. Colour codes: Colour codes: Ni, green; $\mathrm{C}$, gray; $\mathrm{O}$, red 
TABLE-1

SELECTED BOND LENGTHS (̊̊) AND BOND ANGLES (deg) FOR 1

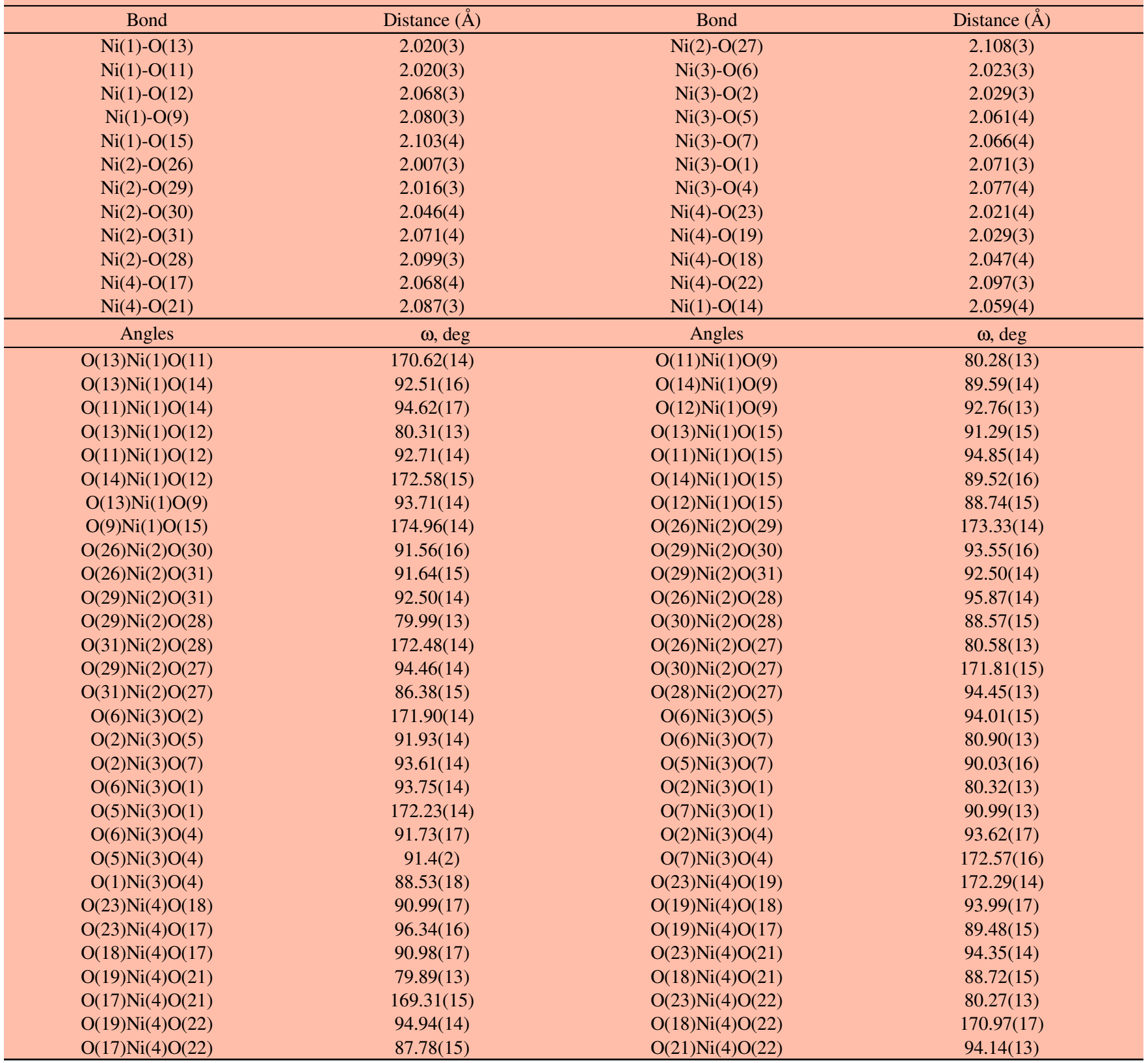

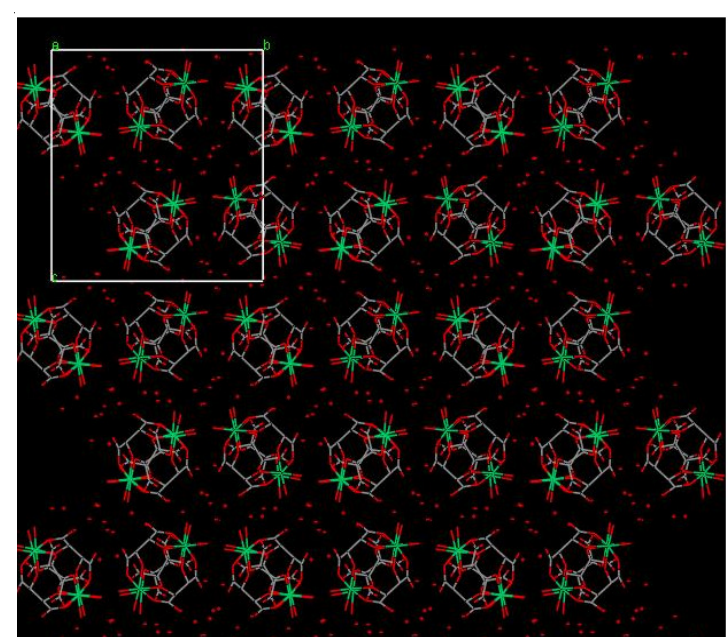

Fig. 4. Molecular packing structure of $\mathbf{1}$ viewed along the a axis. Colour codes: $\mathrm{Ni}$, green; $\mathrm{C}$, gray; $\mathrm{O}$, red
To our best of knowledge, the mutually perpendicular bicycloshape tetranuclear complex featuring Pisces mode is still relatively rare. Selected bond distances and bond angles are shown in Table-1. The hydrogen binding of complex $\mathbf{1}$ is detailed in Table-2.

The IR spectral data show features attributable to the carboxylate and the hydroxy stretching vibrations of the complex 1. The two absorption bands in the regions 35343200 and centered at 1640 and $596 \mathrm{~cm}^{-1}$ corresponding to the $\mathrm{O}-\mathrm{H}$ stretch of the free water and coordinate water ligands, respectively ${ }^{14}$. The strong peaks occurring at 1631 and 1526 $\mathrm{cm}^{-1}$ indicates the antisymmetric and symmetric stretching vibrations of carboxylate. The separation $(\Delta)$ between masym $(\mathrm{COO})$ and msym(COO) is $212 \mathrm{~cm}^{-1}$ which are more than $200 \mathrm{~cm}^{-1}$, suggesting the carboxylate groups coordinate to metal atoms in mono-dentate mode ${ }^{15}$. These results are in agreement with the single-crystal X-ray analysis. 
TABLE-2

DISTANCE (nm) AND ANGLES OF HYDROGEN-BONDING FOR COMPLEX 1

\begin{tabular}{|c|c|c|c|c|}
\hline D-H & $\mathrm{d}(\mathrm{D}-\mathrm{H})$ & $\mathrm{d}(\mathrm{H} . . \mathrm{A})$ & $\angle \mathrm{DHA}$ & $\mathrm{d}(\mathrm{D} . . \mathrm{A})$ \\
\hline $\mathrm{O}(1 \mathrm{~W})-\mathrm{H}(1) \ldots \mathrm{O}(8 \mathrm{~W})$ & 0.81 & 1.91 & 173 & $2.720(6)$ \\
\hline $\mathrm{O}(2 \mathrm{~W})-\mathrm{H}(2) \ldots \mathrm{O}(23)^{\mathrm{i}}$ & 0.81 & 2.04 & 155 & $2.793(5)$ \\
\hline $\mathrm{O}(3 \mathrm{~W})-\mathrm{H}(3) \ldots \mathrm{O}(11)$ & 0.79 & 2.02 & 165 & $2.788(6)$ \\
\hline $\mathrm{O}(4 \mathrm{~W})-\mathrm{H}(4) \ldots \mathrm{O}(20)$ & 0.81 & 2.03 & 155 & 2.792(5) \\
\hline $\mathrm{O}(5 \mathrm{~W})-\mathrm{H}(5) \ldots \mathrm{O}(2)$ & 0.76 & 2.00 & 168 & $2.747(6)$ \\
\hline $\mathrm{O}(5 \mathrm{~W})-\mathrm{H}(6) \ldots \mathrm{O}(26)^{\mathrm{ii}}$ & 0.84 & 1.94 & 171 & $2.779(5)$ \\
\hline $\mathrm{O}(6 \mathrm{~W})-\mathrm{H}(7) \ldots \mathrm{O}(32)^{\mathrm{iii}}$ & 0.78 & 2.01 & 161 & $2.765(5)$ \\
\hline $\mathrm{O}(8 \mathrm{~W})-\mathrm{H}(8) \ldots \mathrm{O}(4 \mathrm{~W})$ & 0.73 & 2.08 & 174 & $2.813(6)$ \\
\hline
\end{tabular}

\section{ACKNOWLEDGEMENTS}

The authors thank the research grant of Phytochemistry Key Laboratory of Shaanxi Province (No.11JS006,11JS007) and the Scientific Research Fund of Shaanxi Provincial Education Department (No. 11JK0603)

\section{REFERENCES}

1. B. Moulton and M.J. Zaworotko, Chem. Rev., 101, 1629 (2001).

2. M.W. Hosseini, Acc. Chem. Res., 38, 313 (2005).

3. A.Y. Robin and K.M. Fromm, Coord. Chem. Rev., 250, 2127 (2006).

4. G.D. Feng, L. Jiang and Z.M. Su, Z. Anorg. Allg. Chem., 637, 2211 (2011).

5. N.L. Rosi, M. Eddaoudi, J. Kim, M. O'Keeffe and O.M. Yaghi, Angew. Chem. Int. Ed. Engl., 41, 284 (2002).

6. C. Serre, F. Millange, C. Thouvenot, N. Gardant, F. Pelle and G. Ferey, J. Mater. Chem., 14, 1540 (2004).
7. Y. Cui, H.L. Ngo, P.S. White and W. Lin, Chem. Commun., 994 (2003).

8. F. Fu, D.S. Li, J. Zhao and W.W. Dong, Chinese J. Struct. Chem., 28, 925 (2009).

9. F. Luo, Y.- Che and J.- Zheng, Inorg. Chem. Commun., 9, 1045 (2006).

10. T. Higashi, Program for Absorption Correction, Rigaku Corporation, Tokyo, Japan (1995).

11. SHELXTL, Version 5.1, Siemens Industrial Automation, Inc. (1997).

12. G.M. Sheldrick, SHELXL-97, Program for the Refinement of Crystal Structure, University of Göttingen, Germany (1997).

13. Y. Wang, G.X. Liu, Y.C. Chen, K.B. Wang and S.-G. Meng, Inorg. Chim. Acta, 363, 2668 (2010).

14. D.S. Li, Y.Y. Wang, X.J. Luan, P. Liu, C.H. Zhou, H.R. Ma and Q.Z. Shi, Eur. J. Inorg. Chem., 2005, 2678 (2005).

15. W. Shi, X.Y. Chen, B. Zhao, A. Yu, H.B. Song, P. Cheng, H.G. Wang, D.Z. Liao and S.P. Yan, Inorg. Chem., 45, 3949 (2006). 